\title{
BMJ
}

\section{Community falls prevention for people who call an emergency ambulance after a fall: randomised controlled trial}

\author{
Philippa A Logan, postdoctoral researcher and occupational therapist, ${ }^{1}$ C A C Coupland, associate professor in \\ medical statistics, ${ }^{1}$ I R F Gladman, professor of older persons medicine and community geriatrician, ${ }^{1}$ O Sahota, \\ professor of orthogeriatric medicine and consultant physician, ${ }^{2}$ V Stoner-Hobbs, triage nurse, ${ }^{3} \mathrm{~K}$ Robertson, \\ community occupational therapist, ${ }^{4} \mathrm{~V}$ Tomlinson, data analyst, ${ }^{3} \mathrm{M}$ Ward, community nurse, ${ }^{5} \mathrm{~T}$ Sach, senior \\ lecturer in health economics, ${ }^{6} \mathrm{~A}$ J Avery, professor of primary care and general practitioner ${ }^{1}$
}

School of Community Health Sciences, University of Nottingham ${ }^{2}$ Nottingham University Hospital, Nottingham

${ }^{3}$ East Midlands Ambulance Service Headquarters, Nottingham

${ }^{4}$ Community Rehabilitation, Lings Bar Hospital, Nottinghamshire Community Health, Nottingham

${ }^{5}$ Community Rehabilitation, Nottingham City Primary Care Trust, Nottingham

${ }^{6}$ Health Economics Group, School of Medicine, Health Policy and

Practice, University of East Anglia, Norwich

Correspondence to: PA Logan, Division of Rehabilitation and Ageing, University of Nottingham, Queen's Medical Centre, Nottingham NG7 2UH

Pip.logan@nottingham.ac.uk

Cite this as: $B M J$ 2010;340:c2102 doi:10.1136/bmi.c2102

\section{ABSTRACT}

Objective To evaluate whether a service to prevent falls in the community would help reduce the rate of falls in older people who call an emergency ambulance when they fall but are not taken to hospital.

Design Randomised controlled trial.

Setting Community covered by four primary care trusts, England.

Participants 204 adults aged more than 60 living at home or in residential care who had fallen and called an emergency ambulance but were not taken to hospital. Interventions Referral to community fall prevention services or standard medical and social care.

Main outcome measures The primary outcome was the rate of falls over 12 months, ascertained from monthly diaries. Secondary outcomes were scores on the Barthel index, Nottingham extended activities of daily living scale, and falls efficacy scale at baseline and by postal questionnaire at 12 months. Analysis was by intention to treat.

Results 102 people were allocated to each group. 99 (97\%) participants in the intervention group received the intervention. Falls diaries were analysed for 88.6 person years in the intervention group and 84.5 person years in the control group. The incidence rates of falls per year were 3.46 in the intervention group and 7.68 in the control group (incidence rate ratio $0.45,95 \%$ confidence interval 0.35 to 0.58 , P 0.001$)$. The intervention group achieved higher scores on the Barthel index and Nottingham extended activities of daily living and lower scores on the falls efficacy scale (all P<0.05) at the 12 month follow-up. The number of times an emergency ambulance was called because of a fall was significantly different during followup (incidence rate ratio $0.60,95 \%$ confidence interval 0.40 to $0.92, P=0.018$ ).

Conclusion A service to prevent falls in the community reduced the fall rate and improved clinical outcome in the high risk group of older people who call an emergency ambulance after a fall but are not taken to hospital.
Trial registration Current Controlled Trials ISRCTN67535605.

\section{INTRODUCTION}

Falls are a common and serious problem in older people. One in four adults aged over $70^{1}$ fall each year and nearly one in two are aged over 80 , half of whom fall again in the following year. ${ }^{23}$ Ambulance services are often called as an emergency to assist older people who have fallen. ${ }^{4}$ In some countries all people who call an ambulance are taken to hospital but in others, such as the United Kingdom and United States, ${ }^{4-6}$ between $30 \%$ and $50 \%$ of such people are not taken to hospital. An emergency ambulance crew will assess the extent of injury and need for acute medical care, but this service will not assess the underlying risk factors for falling nor attempt to ameliorate them. The policies of the ambulance service ${ }^{7}$ in the United Kingdom encourage an increase in the proportion of such people managed at home to reduce demand on hospital emergency departments.

A Cochrane review ${ }^{8}$ showed that multifactorial interventions to prevent falls significantly reduce the rate of falls, and another systematic review ${ }^{9}$ concluded that high intensity interventions that tackle risk factors (rather than simply the provision of information) may be more effective. Despite these interventions, it is not routine for people who fall and who are seen by the ambulance service to be referred to a falls prevention service. We evaluated the use of a rehabilitation service to prevent falls in the community for older people who had called an ambulance because of a fall but had not been taken to hospital.

\section{METHOD}

Adults were eligible for inclusion in the study if they were aged over 60, lived at home or in a care home in one of four primary care trust areas (population 639789 ) in Nottinghamshire, United Kingdom, and had contacted the East Midlands Ambulance Service 
through the emergency telephone system because of a fall but had not been taken to hospital. The four primary care trusts were Nottingham City, Rushcliffe, Broxtowe and Hucknall, and Gedling, which cover city, suburban, and rural addresses. We identified potential participants from ambulance service records and invited them by post to take part in the study. A researcher (PAL) visited respondents at home to explain the study, obtain written informed consent, and collect baseline data. People were excluded if they were unable to give consent, were deemed too ill to participate (for example, terminally ill), or already in a falls prevention rehabilitation programme.

\section{Intervention}

The intervention was provided by four community fall teams, which included occupational therapists, physiotherapists, and nurses. An individualised multifactorial intervention programme was undertaken. This followed the UK clinical fall guidelines ${ }^{10}$ in which participants and therapists set treatment goals. Intervention was primarily delivered in the participants' homes, but the participants were also offered group sessions in community centres.

The interventions at home included training in strength and balance for at least six sessions led by the physiotherapist; an assessment of hazards in the home and modifications to the environment, including provision of equipment such as chair raisers, minor adaptations such as grab handles, and advice, such as removal of items from the floor and improved lighting; and practice in getting up from the floor (provided by the occupational therapists). The nurse completed a review of drugs and blood pressure readings. As required, the participants were referred to other agencies such as the family doctor for a medical review, or social care for help at home. The same fall prevention team also provided an established rolling programme of 12 group sessions on fall prevention, twice weekly over six weeks, in local community centres. Each session lasted two hours, including one hour of muscle strengthening and balance training led by a physiotherapist and one hour of education and functional activities led by an occupational therapist. Sessions also covered advice on nutrition, pacing, strategies for coping with activities of daily living, hazards in the home, equipment, footwear, and how to get up from the floor.

Participants received as many sessions in their own homes as deemed clinically necessary and attended as many group sessions in the rolling programme as they wished, up to a maximum of 12 . The number of techniques used, their duration, and type was recorded for both the home and the group sessions. Participants allocated to the control group had no further study intervention after recruitment and were advised by letter to use existing social and medical services as usual.

\section{Objectives and outcomes}

We hypothesised that rehabilitation for falls prevention would reduce the rate of falls over 12 months compared with usual practice.
Data collected at baseline by questionnaire administered by a researcher in the participants' homes included sex; date of birth; number of falls in the three months before recruitment; the Barthel activities of daily living index,${ }^{11}$ to measure personal ability with activities of daily living; the Nottingham extended activities of daily living scale, ${ }^{12}$ to measure ability with instrumental activities of daily living; and the falls efficacy scale, ${ }^{13}$ to measure fear of falling.

The primary outcome measure was the rate of falls over 12 months, calculated using the number of falls reported by each participant as the numerator and their follow-up time as the denominator. Data on falls were recorded monthly using a diary. ${ }^{14}$ Participants were sent a diary by post each month with a stamped addressed envelope to return the completed previous month's diary. If diaries were not returned researchers masked to group allocation used telephone prompts. The researcher showed participants at the time of recruitment how to complete the diaries and discussed the Prevention of Falls Network Europe ${ }^{15}$ definition of a fall "an unexpected event in which the participant comes to rest on the ground, floor, or lower level." The participants were reminded to include every time that they had a slip or trip in which they lost their balance and landed on the floor, ground, or lower level.

Secondary outcome measures were the time to a first fall within 12 months and whether the participant had or had not fallen at least once during the 12 months of follow-up. In addition to the monthly diaries, participants were sent a questionnaire by post at 12 months to obtain information on other secondary outcome measures: the Barthel activities of daily living index,${ }^{11}$ the Nottingham extended activities of daily living scale, ${ }^{12}$ and the falls efficacy scale. ${ }^{13} \mathrm{~A}$ trained assessor who was independent of the community fall team and masked to group allocation contacted by telephone or visited those participants who did not return their diaries or questionnaires. To examine the potential for assessor bias, we asked assessors to indicate if they were aware of the group to which the participant had been allocated. Two researchers checked and double entered the data on to a database. A third researcher checked all the diary data.

Additional secondary outcome measures were the number of hospital admissions, the number of days in hospital, and any fall related fracture over 12 months. To determine these outcomes a researcher blind to allocation checked the Nottingham University Hospital computer system. The East Midlands Ambulance Service computer system was also checked to determine the number of emergency ambulance calls received for falls over 12 months and the number of such participants taken to an accident and emergency department or left at home.

\section{Sample size}

We determined that to detect a $35 \%$ reduction in the rate of falls from an expected rate of 2.0 falls per year to 1.3 falls per year with $80 \%$ power and $5 \%$ significance 
(two sided), and assuming an overdispersion of 1.5, we needed a sample size of 160 participants $(80$ in each arm). Allowing for 20\% dropout, we set a recruitment target of 200 people (100 in each arm).

\section{Randomisation}

Before the study started, the Nottingham Clinical Trials Unit produced a computer generated randomisation scheme with stratification by primary care trust. The allocation sequence was concealed until allocation. After written consent had been obtained, PAL accessed the randomisation sequence through the internet and assigned the participants to their group. Participants had an equal chance of being assigned to the intervention group (referral to the falls prevention rehabilitation service) or the control group (standard care). On the day of randomisation PAL made a referral to the falls rehabilitation team by telephone who then dealt with the referral in their normal manner. Participants in the intervention group were informed by letter that they would be approached by their local falls prevention team.

\section{Blinding}

It was not possible to blind the participants and treating therapists to allocation group as they would be aware of

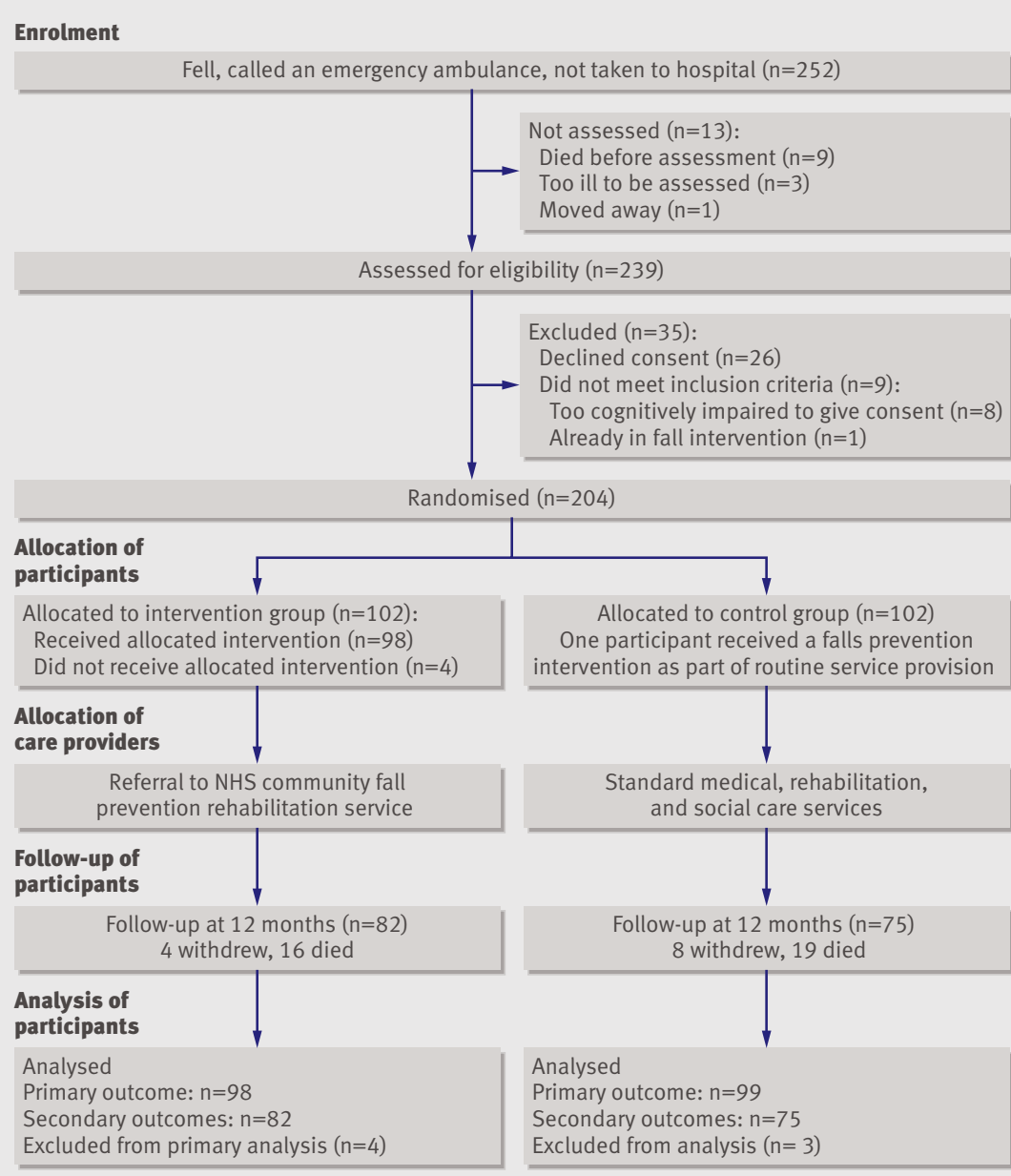

Fig 1| Flow of participants through trial receiving or giving falls rehabilitation. The assessors who contacted the participants to collect missing data on outcome measures and the research staff who input data were blinded to allocation group.

\section{Statistical analysis}

We carried out the analyses according to a prespecified statistical analysis plan. Participants were analysed on an intention to treat basis - that is, according to their allocated group, irrespective of intervention received.

We used descriptive statistics to compare baseline data. The incidence rate ratio of falls comparing the rate of falls between the two groups was estimated using negative binomial regression models. These models analysed the total number of falls reported by each participant allowing for variable lengths of followup up to a maximum of 12 months. Patients were included assuming that each completed falls diary covered 30 days or until they died, withdrew from the study, or reached the end of the 12 month follow-up. The primary analyses only included adjustment for primary care trust. Additional models adjusted for sex, age (61-74, $\geq 75)$, drug use (taking more than four drugs at baseline: yes or no), falls in previous three months at baseline (only the index fall, $\geq 2$ ), and residential status (living at home alone, living at home with others, living in a care home or hospital). We checked residuals and influential points. Tests for interaction were also carried out between the intervention group and age (61-74, $\geq 75)$ and number of falls in the previous three months (only the index fall, $\geq 2$ ).

We used a Cox proportional hazards model to analyse time to first fall and a log-minus-log plot to check the assumption of proportional hazards. The proportions with at least one fall during follow-up were compared using a log-binomial regression model to estimate a risk ratio. We compared the Barthel index, the Nottingham extended activities of daily living, and falls efficacy scale using linear regression or logistic regression, splitting the outcome at the median if the assumptions of linear regression were not valid, and adjusting for primary care trust and baseline values of each respective variable. Using negative binomial regression, adjusting for primary care trust and accounting for length of follow-up, we analysed the number of hospital admissions, the number of days in hospital, and the number of emergency ambulance calls received for falls over 12 months. We analysed falls related fractures over 12 months using Cox proportional hazards model based on time to first fall related fracture resulting in hospital admission during follow-up.

\section{RESULTS}

Overall, 240 participants were recruited (102 in each group) over 16 months from September 2005 (fig 1). The baseline characteristics of the participants were broadly similar between the two groups, although fewer people in the intervention group had a history of more than two falls in the previous three months, 
Table 1|Baseline characteristics of participants randomised to community fall prevention service or to standard medical and social care (control). Values are numbers (percentages) unless stated otherwise

\begin{tabular}{lcc} 
Characteristics & $\begin{array}{c}\text { Control group } \\
(\mathrm{n}=102)\end{array}$ & $\begin{array}{c}\text { Intervention group } \\
(\mathrm{n}=102)\end{array}$ \\
$\begin{array}{l}\text { Men } \\
\text { Women }\end{array}$ & $65(36)$ & $35(34)$ \\
\hline Median (interquartile range) age (years) & $82(78-86)$ & $83(77-86)$ \\
\hline Falls in past 3 months: & $29(28)$ & $28(28)$ \\
\hline 1 (index fall) & $20(20)$ & $29(28)$ \\
\hline 2 & $53(52)$ & $45(44)$ \\
\hline >2 & $61(60)$ & $55(54)$ \\
\hline Taking >4 drugs & $67(66)$ & $57(56)$ \\
\hline Residential status: & $30(29)$ & $39(38)$ \\
\hline Home alone & $5(5)$ & $6(6)$ \\
\hline Home with others & $15(13-18)$ & $15(12-17)$ \\
\hline Care home or hospital & & $14.4(4.18)$ \\
\hline $\begin{array}{l}\text { Median (interquartile range) Barthel activities of daily living index* } \\
\text { (0-20) }\end{array}$ & $6.0(3-9)$ \\
\hline Mean (SD) median Barthel activities of daily living index* (0-20) & $14.8(4.19)$ & $72.0(51-84)$ \\
\hline $\begin{array}{l}\text { Median (interquartile range) Nottingham extended activities of } \\
\text { daily living scale* (0-22) }\end{array}$ & $8.5(4-12)$ & \\
\hline Median (interquartile range) falls efficacy scale† (0-100) & $63.0(47-79)$ & \\
\hline *Higher score indicates greater level of independence in activities. & & \\
\hline Lower score indicates less fear about falling when completing activities. & & \\
\hline
\end{tabular}

were taking more than four drugs, and lived alone (table 1).

\section{Implementation of the intervention}

Four of the 102 participants in the intervention group were not assessed by the falls prevention team: three declined assessment and one died before assessment. Six of the remaining 98 did not require ongoing treatment after assessment but were provided with information and referred to other services such as care at home. Of the 98 participants who were treated, $79(80 \%)$ received intervention purely at home and $19(20 \%)$ had intervention at home and also attended group sessions. The median delay from randomisation to start of treatment was 12 days (interquartile range 4-26 days), the mean number of home or group sessions was 9.9 (SD 8.8), and the median duration of contact time for face to face therapy was 490 minutes (interquartile range 250-1257 minutes). Seventy three participants received seven or more therapy sessions. Participants received a median of eight muscle strengthening sessions (interquartile range 6-12 sessions), 7.5 balance training (interquartile range 2-12) sessions, and 13.5 sessions on functional activities and reduction of hazards (interquartile range 6-18 sessions). The web extra illustrates the frequency of use of common interventions. Only one participant in the control group received a falls prevention programme using existing clinical services.

\section{Primary outcome}

In total, 197 participants (98 in intervention group, 99 in control group) returned one or more falls diaries, and 155 participants (80 in intervention group, 75 in control group) returned all 12 diaries. The number of diaries returned between the two groups did not differ significantly $(\mathrm{P}=0.36$, Mann-Whitney $\mathrm{U}$ test). Three participants in the control group and four in the intervention group did not return any diaries and these participants were excluded from all the falls analyses. The remaining participants were included in the analyses of falls rates using information from any completed diaries.

Overall, 956 falls were reported over the follow-up period, of which 649 were in the control group (spanning 84.5 person years) and 307 in the intervention group (spanning 88.6 person years). Figure 2 shows the rates of falls in the two groups during follow-up. The overall incidence of falls in the intervention group was 3.46 per person years compared with 7.68 per person years in the control group (table 2). The incidence rate ratio, adjusted for primary care trust, was $0.45(95 \%$ confidence interval 0.35 to 0.58 , $\mathrm{P}<0.001)$. No interactions between the intervention and either age or number of previous falls were significant. Results were similar when adjusted for sex, age, drug use, previous falls, residential status, and primary care trust $(0.44,0.34$ to 0.56$)$, and when three participants with the largest residuals were removed $(0.47$, 0.37 to 0.59$)$.

\section{Secondary outcomes}

The questionnaire at 12 months was completed by 157 participants (82 in intervention group, 75 in control group). In 46 of these cases an independent assessor assisted. The assessor correctly guessed the allocation group for seven $(15 \%)$ cases. Hospital data were complete for 202 participants and data on ambulance use were complete for 203 participants. Table 2 shows the
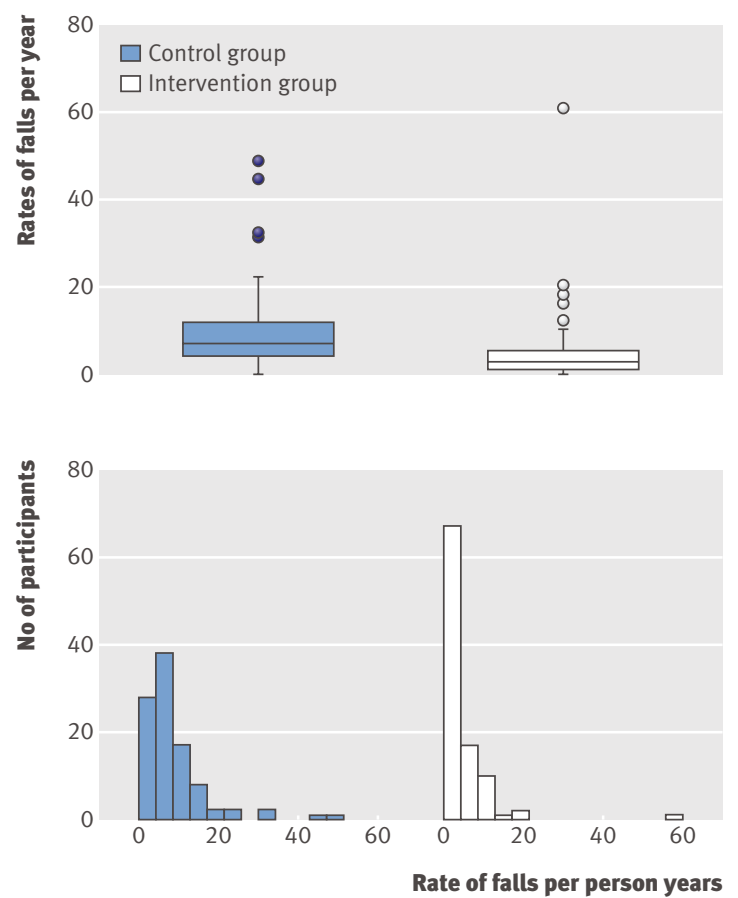

Fig 2 | Rate of falls by treatment group 
Table 2 |Outcome measures by allocation group. Values are numbers (percentages) unless stated otherwise

\begin{tabular}{|c|c|c|c|c|}
\hline Outcome measures & $\begin{array}{l}\text { Control group } \\
\quad(n=102)\end{array}$ & $\begin{array}{l}\text { Intervention group } \\
\qquad(n=102)\end{array}$ & $\begin{array}{c}\text { Effect size* } \\
(95 \% \mathrm{Cl})\end{array}$ & $P$ value \\
\hline \multicolumn{5}{|l|}{ Primary outcome: } \\
\hline Rate of falls per person year & 7.68 & 3.46 & $0.45 \dagger(0.35$ to 0.58$)$ & $<0.001$ \\
\hline \multicolumn{5}{|l|}{ Secondary outcomes: } \\
\hline$\geq 1$ falls during follow-up & $96(97)$ & $81(83)$ & $0.86 \ddagger(0.78$ to 0.94$)$ & 0.001 \\
\hline Median (interquartile range) time to first fall (days) & $21(10-90)$ & $166(34-297)$ & $0.32 \S(0.23$ to 0.44$)$ & $<0.001$ \\
\hline$\geq 1$ fractures admitted to hospital & $6(6)$ & $3(3)$ & $0.51 \S(0.13$ to 2.06$)$ & 0.35 \\
\hline Died by 12 month assessment & $16(16)$ & $14(14)$ & $0.89 \S(0.43$ to 1.82$)$ & 0.74 \\
\hline$\geq 1$ admissions to hospital & $54(53)$ & $53(52)$ & $0.98 \rrbracket(0.56$ to 1.70$)$ & 0.93 \\
\hline Total No of hospital admissions & 99 & 97 & $0.98 \dagger(0.69$ to 1.40$)$ & 0.93 \\
\hline Total No of days in hospital in year & 1141 & 1257 & $1.13 \dagger(0.60$ to 2.13$)$ & 0.70 \\
\hline Total No of times emergency ambulance called owing to fall & 365 & 245 & $0.60 \dagger(0.40$ to 0.92$)$ & 0.018 \\
\hline Median (interquartile range) Barthel activities of daily living index (0-20) & $15(12-17)$ & $15(12-18)$ & $2.91 \rrbracket(1.18$ to 7.20$)$ & 0.021 \\
\hline Median (interquartile range) Nottingham extended activities of daily living scale (0-22) & $6(1-10)$ & $8(4-13)$ & $3.47^{\star \star}(2.13$ to 4.81$)$ & $<0.001$ \\
\hline Median (interquartile range) falls efficacy scale†† (0-100) & $76(53-91)$ & $57(41-75)$ & $-16.5^{\star *}(-23.2$ to -9.8$)$ & $<0.001$ \\
\hline \multicolumn{5}{|l|}{ 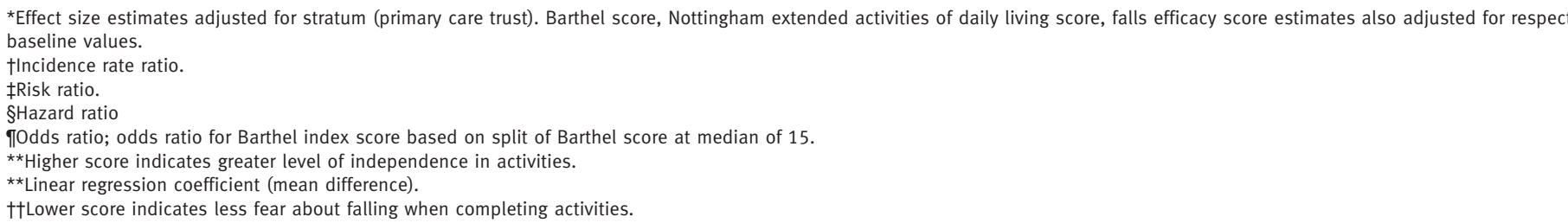 } \\
\hline
\end{tabular}

results for the secondary outcomes. Benefits were significant for participants in the intervention group compared with the control group: higher scores on the Barthel index and Nottingham extended activities of daily living and lower scores on the falls efficacy scale after adjusting for their respective baseline values. We also had two objective indicators related to falls during follow-up - the number of participants admitted to hospital with a fracture and number of times an emergency ambulance was called because of a fall. Both of these were established from service databases rather than self reported by participants. Both indicators were less frequent in the intervention group (table 2), with a significant difference for the number of times an emergency ambulance was called because of a fall; the number of fractures was, however, small. This is consistent with the lower rate of falls as reported by participants.

In the intervention group 81 participants (83\%) reported one or more falls over the follow-up period

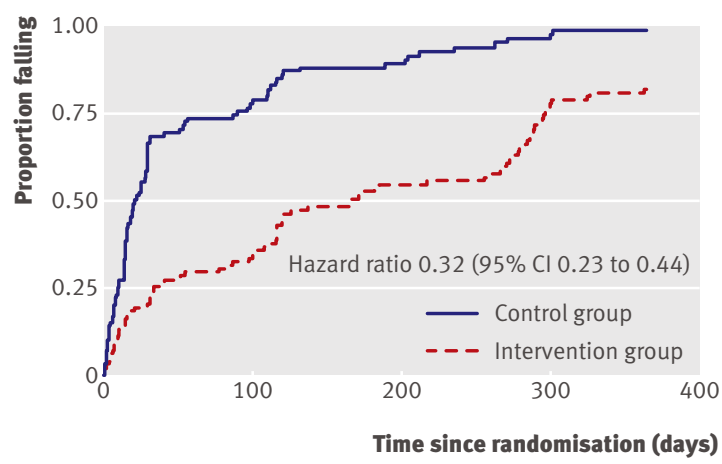

Fig 3 Kaplan-Meier curve for time to first fall by treatment group compared with $96(97 \%)$ in the control group (risk ratio $0.86,95 \%$ confidence interval 0.78 to $0.94, \mathrm{P}=0.001$ ). The median time to the first fall during follow-up was 166 days in the intervention group and 21 days in the control group. Figure 3 shows the Kaplan-Meier plot for the time to first fall. The hazard ratio from the Cox regression model was 0.32 (95\% confidence interval 0.23 to 0.44 ) adjusted for primary care trust.

\section{DISCUSSION}

A rehabilitation service to prevent falls in the community for older people ( $>60$ years) was associated with a sizeable reduction in the rate of falls $(55 \%)$ over the subsequent year. This effect was larger than we had anticipated and larger than the pooled effect $(25 \%)$ seen in the Cochrane review of studies on fall prevention. ${ }^{8}$ Our population was at high risk of falls as evidenced by participants reporting an average of two falls in the three months before recruitment. The participants had functional limitations, with a median Barthel index score of 15.0 at baseline (mean 14.2). Our population is different from those in other studies of similar interventions where participants were more functionally able at baseline: mean Barthel index score of $18.7^{16}$ and 19.0. ${ }^{17}$ During the 12 months' follow-up 7.7 falls per year occurred in the control group, compared with 3.5 per year in the intervention group. We believe this reduction to be clinically important.

\section{Strengths and limitations of the study}

We do not think that allocation bias can explain this major difference, as we used an independent, telephone randomisation system after obtaining informed consent, and the baseline characteristics of the groups were broadly similar. Also, the findings are unlikely to 


\section{WHAT IS ALREADY KNOWN ON THIS TOPIC}

Many people fall and call an ambulance but are not taken to hospital

\section{WHAT THIS STUDY ADDS}

A community based service to prevent falls reduced the number of falls over a year in older people ( 260 years) who had called an emergency ambulance owing to a fall but not been taken to hospital

The service also led to increased levels of activities of daily living and reduced fear of falling

be due to observer bias as outcomes were largely obtained by postal questionnaire and the assessors, when used, were masked to allocation and were only able to guess the correct group 15\% of the time. The data were checked by two researchers and double entered onto the computer. However, some degree of respondent bias is possible as the participants were not masked to the intervention. We used monthly falls diaries to ascertain the number of falls occurring during follow-up, having explained to participants at recruitment how to complete the diaries. Unlike monitoring technology, the diaries are inexpensive and nonintrusive. ${ }^{3}$ They reduce recall bias in the estimation of falls compared with attempting to ascertain falls at final follow-up. ${ }^{14}$ Ascertainment by post, as opposed to by direct contact, avoids observer bias. Nevertheless, it is possible that residual bias could remain by intervention participants either over-reporting or underreporting falls. Within the financial and time restriction of a study, however, we believe that falls diaries are the best way to measure the rate of falls.

To establish the plausibility of a genuine treatment effect we examined the amount of treatment given and found that over $79 \%$ of treated participants received at least seven or more intervention sessions. This is in line with the recommendations made by falls clinical guidelines ${ }^{10}$ and confirms other studies that have found positive results in selected high risk participants, such as those who have already fallen when given an optimal amount of treatment. ${ }^{1819}$ Other people who fall may equally benefit from this type of intervention after an emergency ambulance call-for example, those with cognitive impairment, who we did not include because they did not have capacity to consent, and those living in residential homes who may not have seen the invitation to take part in the study.

Importantly, we saw improvements in activities of daily living in the intervention group (higher scores on the Barthel index and Nottingham extended activities of daily living) and a reduction in fear of falling (falls efficacy scale). We assume that this was due to the package of intervention delivered by the falls prevention services, although we cannot specify which components were particularly effective. Restrictive practices that reduce the risk of falls by limiting activity do not seem to have achieved a reduction in the number of falls. Commensurate with a reduction in the number of falls there was a reduction in the number of times an ambulance was called for recurrent falls.
Half of the participants had one or more hospital admissions but the rates between groups did not differ.

\section{Conclusion}

People who have fallen and called an emergency ambulance but are not taken to hospital are at high risk of falling again. This group are unlikely in usual clinical practice to receive formal falls prevention interventions. Immediate referral of such people to a falls prevention rehabilitation service may reduce the number of further falls and improve other indicators of health.

A study of the economic consequences of these clinical findings is required. A multicentre study would help to establish generalisability and a larger study could have the power to determine the effect of the intervention on costly outcomes such as fractures and admissions to hospital. In such a study, consideration should be given to an objective measure of physical activity and falls.

We thank CL Fellows (clinical audit manager, East Midlands Ambulance Service), who initially contacted participants through the ambulance service, and C Simms-Jones (clinical audit office), who helped collect data from the ambulance service

Contributors: PAL (principal investigator) designed the protocol, secured funding, recruited participants, collected and analysed data, and wrote the paper. She is guarantor. CACC analysed the data, interpreted the results, and drafted sections of the paper. JRFG designed and wrote the protocol and grant application, interpreted and analysed the results, and wrote the paper. OS designed and wrote the protocol and grant application, interpreted the results, and wrote the paper. VS-H designed and wrote the protocol and grant application, collected data, and wrote the paper. KR designed and wrote the protocol and grant application, collected and checked the data, and wrote the paper. VT designed and wrote the protocol and grant application; collected, checked, and input the data; and wrote the paper. MW designed and wrote the protocol and grant application, collected and checked the data, interpreted the results, and wrote the paper. TS designed and wrote the protocol and grant application, interpreted the results, and wrote the paper. AJA designed and wrote the protocol and grant application, interpreted the results, and wrote the paper. All authors, external and internal, had full access to all of the data (including statistical reports and tables) in the study and can take responsibility for the integrity of the data and the accuracy of the data analysis

Funding: This study was funded by a postdoctoral training scholarship awarded to PAL from the UK NHS National Institute of Health Research. The funding source provided an external peer review of the study protocol but did not have any role in the data collection, analysis, report writing, or decision to publish this paper.

Competing interests: All authors have completed the unified competing interest form at www.icmje.org/coi_disclosure.pdf (available on request from the corresponding author) and declare (1) no financial support for the submitted work from anyone other than their employer; (2) no financial relationships with commercial entities that might have an interest in the submitted work; (3) no spouses, partners, or children with relationships with commercial entities that might have an interest in the submitted work; and (4) no non-financial interests that may be relevant to the submitted work.

Ethical approval: This study was approved by the North Nottinghamshire local research ethics committee and the Nottinghamshire primary care trusts for NHS research and development committee (05/Q2402/53). Data sharing: The technical appendix, statistical code, and dataset are available from the corresponding author at pip.logan@nottingham.ac.uk.

1 Sayer AASH, Martin HJ, Dennison EM, Anderson FH, Cooper C. Falls, sarcopenia, and growth in early life: findings from the Hertfordshire Cohort Study. Am J Epidemiol 2006;164:665-71.

2 Tinetti M, Speechley M, Ginter S. Risk factors for falls among elderly persons living within the community. N Engl J Med 1988;319:1701-7 
3 Snijder MB, van Schoor NM, Pluijm SMF, van Dam RM, Visser M, Lips P. Vitamin D status in relation to one-year risk of recurrent falling in older men and women. J Clin Endocrinol Metab 2006;91:2980-5.

4 Marks P, Daniel T, Afolabi O, Spiers G, Nguyen-Van-Tam J. Emergency (999) calls to the ambulance service that do not result in the patients being transported to hospital: an epidemiological study. Emerg Med / 2002;19:449-52.

5 Snooks H, Halter M, Close J, Cheung W, Moore H, Roberts S. Emergency care of older people who fall: a missed opportunity. Qual Saf Health Care 2006;15:390-2.

6 Weiss S, Chong R, Ong M, Ernst AA, Balash M. Emergency medical services screening of elderly falls in the home. Prehosp Emerg Care 2003;7:79-84

7 Department of Health. Taking healthcare to the patient: transforming NHS ambulance services. 2005. www.dh.gov.uk/prod_consum_dh/ groups/dh_digitalassets/@dh/@en/documents/digitalasset/ dh_4114270.pdf.

8 Gillespie LD, Robertson MC, Gillespie WJ, Lamb SE, Gates S, Cumming RG, et al. Interventions for preventing falls in older people living in the community. Cochrane Database Syst Rev 2009;(2):CD007146

9 Gates S, Fisher JD, Cooke MW, Carter YH, Lamb SE. Multifactorial assessment and targeted intervention for preventing falls and injuries among older people in community and emergency care settings: systematic review and meta-analysis. $B M$ 2008;336:130-3.

10 NICE. Falls: the assessment and prevention of falls in older people. 2004. www.nice.org.uk/cg21.
11 Collin C, Wade DT, Davies S, Horne V. The Barthel ADL index: a reliability study. Int Disabil Stud 1988;10:61-3.

12 Gladman JRF, Lincoln NB, Adams SA. Use of the extended ADL scale with stroke patients. Age Ageing 1993;22:419-24.

13 Tinetti ME, Richman D, Powell L. Falls efficacy as a measure of fear of falling. J Gerontol 1990;45:239-43.

14 Cummings SRNM, Kidd S. Forgetting falls. The limited accuracy of recall of falls in the elderly. J Am Geriatr Soc 1988;36:613-6.

15 Lamb S, Jørstad-Stein EC, Hauer K, Becker C, on behalf of the Prevention of Falls Network Europe and Outcomes Consensus Group. Development of a common outcome data set for fall injury prevention trials: the Prevention of Falls Network Europe Consensus. J Am Geriatr Soc 2005;53:1618-23.

16 Close J, Ellis M, Hooper R, Glucksman E, Jackson S, Swift C. Prevention of falls in the elderly trial (PROFET): a randomised controlled trial. Lancet 1999;353:93-7.

17 Lightbody E, Watkins C, Leathley M, Sharma A, Lye M. Evaluation of a nurse-led falls prevention programme versus usual care: a randomized controlled trial. Age Ageing 2002;31:203-10.

18 Clemson L, Cumming RG, Kendig $\mathrm{H}$. The effectiveness of a community-based program for reducing the incidence of falls in the elderly: a randomized trial. J Am Geriatr Soc 2004;52:1487-94.

19 Weatherall M. Prevention of falls and fall-related fractures in community-dwelling older adults: a meta-analysis of estimates of effectiveness based on recent guidelines. Ill Med / 2004;34:102-8.

Accepted: 29 March 2010 